\title{
Perfil sociodemográfico e clínico dos idosos atendidos em uma unidade de pronto atendimento de Minas Gerais
}

\author{
Sociodemographic and clinical profile of the elderly treated in an emergency care unit in \\ Minas Gerais
}

Perfil sociodemográfico y clínico de personas mayores servidas en una unidad lista de Minas Gerais

Amanda Karolina Souza Alkmim ${ }^{1 *}$, Francielle Carolina Santos ${ }^{1}$.

\begin{abstract}
RESUMO
Objetivo: Avaliar o perfil sociodemográfico e clínico dos pacientes idosos atendidos em uma Unidade de Pronto Atendimento. Métodos: Estudo descritivo, com abordagem quantitativa e levantamento de dados secundários de 375 pacientes idosos atendidos no período de um ano. Resultados: A amostra teve predomínio do sexo feminino $(61,6 \%)$ e média de idade de 72,94 anos. As principais queixas foram relacionadas a problemas nos membros $(30,1 \%)$, seguida de dor abdominal $(11,5 \%)$, mal-estar $(10,7 \%)$, dispneia $(9,9 \%)$, dor lombar $(7,5 \%)$ e dor torácica (5,3\%). Apenas 10,9\% foram classificados como pacientes graves. Os principais fluxogramas utilizados foram problemas de extremidades $(26,7 \%)$, seguido de mal-estar no adulto $(10,7 \%)$ e dor abdominal em adultos $(10,7 \%)$. Dor leve recente $(40,5 \%)$, dor moderada $(20,3 \%)$ e edema $(3,2 \%)$ foram os discriminadores mais prevalentes. Após o atendimento médico, $86,4 \%$ receberam alta domiciliar. Conclusão: Foi possível identificar que uma parcela importante dos atendimentos se tratava de atendimentos não urgentes e que, em grande parte, poderiam ser atendidos e solucionados na atenção primária à saúde, o que aponta uma descaracterização do verdadeiro papel das unidades de pronto atendimento.
\end{abstract}

Palavras-chave: Idoso, Perfil de saúde, Serviços de atendimento de emergência, Enfermagem em emergência.

\section{ABSTRACT}

Objective: To evaluate the sociodemographic and clinical profile of elderly patients treated at an Emergency Care Unit. Methods: Descriptive study, with a quantitative approach and survey of secondary data of 375 elderly patients treated over a period of one year. Results: The sample was predominantly female (61.6\%) and had a mean age of 72.94 years. The main complaints were related to limb problems $(30.1 \%)$, followed by abdominal pain $(11.5 \%)$, malaise $(10.7 \%)$, dyspnea $(9.9 \%)$, low back pain $(7,5 \%)$ and chest pain $(5.3 \%)$. Only $10.9 \%$ were classified as critically ill patients. The main flowcharts used were extremity problems $(26.7 \%)$, followed by malaise in adults $(10.7 \%)$ and abdominal pain in adults (10.7\%). Recent mild pain (40.5\%), moderate pain $(20.3 \%)$ and edema (3.2\%) were the most prevalent discriminators. After medical care, $86.4 \%$ were discharged home. Conclusion: It was possible to identify that an important portion of the consultations were non-urgent consultations and that, in large part, they could be attended and resolved in primary health care, which points to a mischaracterization of the real role of the emergency units attendance.

Keywords: Elderly, Health profile, Emergency care services, Emergency nursing.

\section{RESUMEN}

Objetivo: Evaluar el perfil sociodemográfico y clínico de pacientes de edad avanzada tratados en una Unidad de Atención de Emergencia. Métodos: Estudio descriptivo, con un enfoque cuantitativo y encuesta de datos secundarios de 375 pacientes ancianos tratados durante un período de un año. Resultados: La muestra era predominantemente femenina $(61,6 \%)$ y tenía una edad media de 72,94 años. Las principales quejas se relacionaron con problemas en las extremidades (30.1\%), seguidas de dolor abdominal (11.5\%), malestar

\footnotetext{
${ }^{1}$ Hospital Municipal Odilon Behrens, Belo Horizonte - MG. *E-mail: amnda_souza@hotmail.com
} 
(10.7\%), disnea (9.9\%), dolor lumbar $(7,5 \%)$ y dolor en el pecho $(5,3 \%)$. Solo el $10.9 \%$ fueron clasificados como pacientes críticos. Los principales diagramas de flujo utilizados fueron problemas de extremidades $(26.7 \%)$, seguidos de malestar en adultos (10.7\%) y dolor abdominal en adultos (10.7\%). El dolor leve reciente $(40.5 \%)$, el dolor moderado (20.3\%) y el edema (3.2\%) fueron los discriminadores más prevalentes. Después de la atención médica, el $86.4 \%$ fueron dados de alta. Conclusión: Fue posible identificar que una parte importante de la atención era atención no urgente y que, en gran medida, podía ser atendida y resuelta en la atención primaria de salud, lo que apunta a una caracterización errónea del verdadero papel de las unidades de atención de emergencia.

Palabras clave: Ancianos, Perfil de salud, Servicios de atención de emergencia, Enfermería de emergencia.

\section{INTRODUÇÃO}

O aumento da expectativa de vida e do envelhecimento da população tem ocorrido de maneira acelerada nos países em desenvolvimento. $O$ crescimento da população idosa nas últimas décadas é um fenômeno mundial, as pessoas com 60 anos ou mais de idade representam mais de $12 \%$ da população mundial, visto que, até 2030, esse índice aumentará para 16,5\% em todo o mundo. No Brasil estima-se que em 2025 o número será 32 milhões, sendo o sexto país em termos de população idosa (TIENSOLI SD, et al., 2014).

O envelhecimento na atualidade tem se transformado em uma preocupação, pois aliado às limitações fisiológicas resultantes da senescência, há o crescimento das doenças crônicas não transmissíveis, as doenças infecciosas e causas externas, o que caracteriza um cenário de tripla carga de doenças, tornando o idoso cada vez mais dependente de cuidados (OLIVEIRA CP, et al., 2018).

Para melhor atendimento das demandas, sobretudo em termos de saúde e qualidade de vida dos idosos, fez-se necessária a criação de políticas públicas e sociais capazes de amparar essa parcela da população de maneira adequada e estratégica.

Em 2006, o Ministério da Saúde (MS) sancionou a Política Nacional de Saúde à Pessoa Idosa, que visa à promoção, prevenção e recuperação da saúde, pautando-se nas diretrizes do Sistema Único de Saúde (SUS), referindo que a Atenção Primária à Saúde (APS), deve funcionar como porta de entrada preferencial do sistema, articulando-se com os demais níveis de complexidade (OLIVEIRA CP, et al., 2018; RISSARDO LK, et al., 2016).

Importante destacar que o sistema de saúde é organizado em redes de atenção, com pontos de assistência de diferentes densidades tecnológicas e apresentam missão e objetivos comuns, são determinadas sem hierarquia, organizando-se de forma poliárquica, em que todos os pontos de atenção à saúde são do mesmo modo importantes e se relacionam horizontalmente (BRASIL, 2011).

No entanto, a APS exibe alguns desafios que exigem soluções, como por exemplo, a oferta insuficiente de serviços de saúde tendo em vista a carência de recursos humanos e tecnológicos. Circunstância essa que reflete diretamente na demanda de outras instituições de saúde, como as Unidades de Pronto Atendimento (UPA), onde é identificada uma grande procura de pacientes com quadros muitas vezes passíveis de serem resolvidos na atenção primária (RÊGO AS, et al., 2017).

Nesse contexto, faz-se necessária a adequação do atendimento em todos os pontos de atenção, especialmente nos serviços com grande demanda de idosos, como as UPA, que se configuram por estabelecimentos de saúde não hospitalares e concentram atendimentos cuja complexidade é considerada intermediária e que se une ao componente do sistema de atenção à saúde, estabelecido pela Política Nacional de Atenção às Urgências (PNAU) (SCOLARI GAS, et al., 2018).

O conhecimento das características da população que utiliza determinado serviço de emergência torna-se indispensável para a organização das ações em saúde, pois, além de favorecer a diminuição das superlotações nos serviços, viabiliza a prestação de cuidado seguro e com qualidade. Segundo o MS, para que seja possível a organização de uma rede que consiga atender, de maneira eficaz, aos principais problemas de saúde dos indivíduos que necessitem de cuidados na área de urgência e emergência, é preciso levar em consideração quem são, onde estão e quais as necessidades dessa população (BRASIL, 2013). 
Ao analisar a complexidade do processo de envelhecimento, relacionado à alta demanda dos idosos em todos os pontos de atenção, torna-se relevante traçar o perfil da população que frequenta um serviço de emergência. Acredita-se, portanto, que desenvolver pesquisa com esse foco seja imprescindível para subsidiar o planejamento de intervenções nas localidades o que contribui sobremaneira para a redução dos atendimentos evitáveis, principalmente da população idosa (SCOLARI GAS, et al., 2018). Diante do exposto, este estudo teve como objetivo avaliar o perfil sociodemográfico e clínico dos pacientes idosos atendidos em uma Unidade de Pronto Atendimento no município de Belo Horizonte, Minas Gerais.

\section{MÉTODOS}

Trata-se de um estudo descritivo de abordagem quantitativa e delineamento transversal, a partir do levantamento de dados secundários, realizado em uma UPA da regional Noroeste de Belo Horizonte. A cidade de Belo Horizonte está organizada em nove administrações regionais, sendo que cada uma delas contém uma UPA de referência. A UPA em estudo oferta serviços de pronto atendimento 24 horas com clínica médica, cirurgia geral e ortopedia.

A escolha da unidade ocorreu devido inauguração recente, em dezembro de 2015, não havendo, até o momento, conhecimento da demanda de idosos atendida. Sendo assim, considerando a média mensal de 3.074 atendimentos de pacientes idosos, o cálculo da amostra foi estimado em 342 prontuários, com grau de confiabilidade de $95 \%$ e erro amostral de 5\%. Para aumentar a confiabilidade, foram analisados 375 prontuários de pacientes atendidos entre janeiro a dezembro de 2018, em amostragem aleatória simples.

A ordem de prioridade do atendimento, desde a inauguração, era estabelecida através da aplicação do protocolo de Manchester, cuja classificação era embasada em cinco eixos de gravidade por cores (azul: não urgente, verde: pouco urgente, amarelo: urgente, laranja: muito urgente e vermelho: emergência). Neste a classificação ocorre a partir de fluxogramas das principais queixas apresentadas nos serviços de urgência. Cada fluxograma dispõe de um conjunto de discriminadores que caracterizam os sinais e sintomas relacionados à queixa principal anunciada pelo paciente (GONÇALES PC, et al., 2015).

Constituíram a amostra os prontuários de pacientes com idade igual ou superior a 60 anos, que foram atendimentos na UPA da regional noroeste dentro do período analisado e que passaram pela classificação de risco com preenchimento da queixa principal apresentada, fluxo, discriminador e prioridade da classificação de risco obtida ao final da avaliação do enfermeiro.

Para a coleta de dados, foi utilizado o instrumento, desenvolvido para nortear o estudo e que teve como fonte de dados as informações do sistema informatizado da referida UPA, que incluíam dados do perfil demográfico (sexo, idade e procedência), assim como variáveis clínicas (queixa principal e comorbidades), associadas ao acolhimento com classificação de risco (ACCR) (fluxo, discriminador e prioridade) e dados do desfecho clínico (alta, transferência e óbito).

Nas análises foi definido o termo "alta prioridade" para os pacientes classificados no protocolo de Manchester com as cores vermelha e laranja e "baixa prioridade" para as cores amarela, verde e azul (GUEDES HM, et al., 2015). Neste estudo apenas uma pessoa foi classificada na cor azul e devido ao baixo impacto de retirada optou-se por mantê-la nas análises com a classificação em verde. Os dados coletados foram consolidados no programa estatístico informatizado.

As análises estatísticas foram realizadas utilizando o software Statistical Package for Social Sciences (SPSS), versão 15.0 para Windows. As metodologias adotadas foram a análise descritiva a fim de entender o comportamento da amostra e a análise bivariada, empregando o teste $x 2$ de Pearson, considerando um valor de $p \leq 0,05$, com intuito de medir a associação entre a prioridade da classificação de risco e as variáveis independentes.

O estudo desenvolveu-se em conformidade com as diretrizes e normas regulamentadas pela Resolução no 466/2012 do Conselho Nacional de Saúde, sendo que a pesquisa foi submetida à apreciação do Comitê de Ética em Pesquisa com Seres Humanos do Hospital Metropolitano Odilon Behrens, e seu projeto aprovado por meio do parecer no 3.793.482. Por tratar-se de pesquisa nos prontuários dos pacientes foi solicitada a dispensa do Termo de Consentimento Livre e Esclarecido (TCLE). 


\section{RESULTADOS}

Da amostra estudada, 375 participantes, $61,6 \%$ eram do sexo feminino e $38,4 \%$ do sexo masculino. A idade mínima dos pacientes foi de 60 anos e a máxima 99 anos, média de 72,94 anos (Desvio Padrão (DP) $=10,23$ anos). Os pacientes residiam, predominantemente, em Belo Horizonte (88,8\%), e em relação à região de moradia a regional Noroeste foi a mais apontada $(30,4 \%)$. A busca pelo serviço ocorreu, em quase sua totalidade, por demanda espontânea $(92,5 \%)$, sendo apenas $4,5 \%$ encaminhadas pelo Serviço de Atendimento Móvel de Urgência (SAMU) (Tabela 1).

Tabela 1 - Características dos idosos atendidos na UPA Noroeste - MG - 2018.

\begin{tabular}{|c|c|c|}
\hline Variáveis & $\mathbf{n}$ & $\%$ \\
\hline \multicolumn{3}{|l|}{ Sexo } \\
\hline Masculino & 144 & 38,4 \\
\hline Feminino & 231 & 61,6 \\
\hline Total & 375 & 100,0 \\
\hline \multicolumn{3}{|l|}{ Idade (anos) } \\
\hline 60 a 70 anos & 198 & 52,8 \\
\hline 71 a 80 anos & 90 & 24,0 \\
\hline$>81$ & 87 & 23,2 \\
\hline Total & 375 & 100,0 \\
\hline \multicolumn{3}{|l|}{ Município de procedência } \\
\hline Belo Horizonte & 333 & 88,8 \\
\hline Outros & 42 & 11,2 \\
\hline Total & 375 & 100,0 \\
\hline \multicolumn{3}{|l|}{ Regional de procedência } \\
\hline Barreiro & 3 & 0,8 \\
\hline Centro & 7 & 1,9 \\
\hline Leste & 11 & 2,9 \\
\hline Oeste & 14 & 3,7 \\
\hline Norte & 22 & 5,9 \\
\hline Venda Nova & 42 & 11,2 \\
\hline Região Metropolitana & 42 & 11,2 \\
\hline Pampulha & 46 & 12,3 \\
\hline Nordeste & 70 & 18,7 \\
\hline Noroeste & 114 & 30,4 \\
\hline Sem Registro & 4 & 1,0 \\
\hline Total & 375 & 100,0 \\
\hline \multicolumn{3}{|l|}{ Procedência } \\
\hline Encaminhamento & 11 & 3,0 \\
\hline SAMU & 17 & 4,5 \\
\hline Demanda espontânea & 347 & 92,5 \\
\hline Total & 375 & 100,0 \\
\hline
\end{tabular}

Fonte: Alkmim AK e Santos CS, 2020.

A principal queixa reportada pelos pacientes estava relacionada a problemas nos membros $(30,1 \%)$, seguida de dor abdominal (11,5\%) e mal-estar (10,7\%). Quanto à situação clínica, observou-se que $91,0 \%$ referiram possuir algum tipo de doença crônica relatada em prontuário eletrônico, sendo a hipertensão arterial sistêmica $(46,0 \%)$ e a diabetes mellitus $(25,0 \%)$ as mais frequentes. Já em $9,0 \%$ da amostra a informação não foi registrada no sistema.

A Tabela 2 mostra a distribuição da classificação dos pacientes idosos, segundo o nível de risco que é realizado na unidade através do protocolo de Manchester, sendo que 10,9\% foram classificados como pacientes graves (laranja e vermelho). De acordo com o fluxograma de atendimento, o mais utilizado foi o de problemas em extremidades $(26,7 \%)$, seguido de mal-estar no adulto $(10,7 \%)$ e dor abdominal em adultos $(10,7 \%)$. Dor leve recente $(40,5 \%)$, dor moderada $(20,3 \%)$ e edema $(3,2 \%)$ foram os discriminadores mais prevalentes. 
Tabela 2 - Classificação dos pacientes idosos segundo os níveis de risco do Protocolo de Manchester - MG - 2018.

\begin{tabular}{ccc}
\hline Classificação de risco & $\mathbf{N}$ & $\%$ \\
\hline Verde & 199 & 53,1 \\
Amarelo & 135 & 36,0 \\
Laranja & 33 & 8,8 \\
Vermelho & 8 & 2,1 \\
Total & 375 & 100,0 \\
\hline
\end{tabular}

Fonte: Alkmim AKS e Santos CS, 2020.

O tempo de espera entre o registro do paciente e o AACR variou de 0:00 a 0:51 minutos, com mediana de $0: 11$ minutos ( $D P=0: 10$ minutos), sendo que $48,3 \%$ receberam atendimento em até 10 minutos. $O$ tempo decorrente entre a classificação de risco e o primeiro atendimento para cada nível de prioridade clínica está apresentado na Tabela 3. Em média, os pacientes aguardavam 1:05h até a consulta médica.

Tabela 3 - Tempo do primeiro atendimento após a classificação de risco segundo nível de prioridade clínica - MG - 2018.

\begin{tabular}{|c|c|c|c|c|c|c|}
\hline Classificação de risco & & $\mathbf{N}$ & Mínimo & Máximo & Média & Desv. típ. \\
\hline Vermelho & $\begin{array}{l}\text { Tempo de espera para } \\
\text { atendimento médico }\end{array}$ & 8 & 0:00 & 0:00 & 0:00 & $0: 00$ \\
\hline Laranja & $\begin{array}{l}\text { Tempo de espera para } \\
\text { atendimento médico }\end{array}$ & 33 & 0:02 & $0: 20$ & $0: 08$ & $0: 04$ \\
\hline Amarelo & $\begin{array}{l}\text { Tempo de espera para } \\
\text { atendimento médico }\end{array}$ & $\begin{array}{c}13 \\
5\end{array}$ & 0:00 & $1: 36$ & $0: 30$ & $0: 26$ \\
\hline Verde/Azul & $\begin{array}{l}\text { Tempo de espera para } \\
\text { atendimento médico }\end{array}$ & $\begin{array}{c}19 \\
9\end{array}$ & 0:02 & $4: 16$ & $1: 42$ & $1: 08$ \\
\hline
\end{tabular}

Fonte: Alkmim AKS e Santos CS, 2020.

Os atendimentos de clínica médica foram predominantes, representando $76,3 \%$ da amostra. A ortopedia foi responsável por $14,4 \%$ dos atendimentos e a cirurgia geral por 9,3\%. Em relação ao desfecho após o atendimento médico, $86,4 \%$ receberam alta domiciliar, $7,0 \%$ foram transferidos para outras instituições de saúde e $6 \%$ para outros setores. A proporção de óbitos foi de 1,3\%.

As variáveis que tiveram associação significativa com a classificação de risco, utilizando-se a metodologia do Teste Qui-Quadrado, a um nível de significância de até $5 \%$, foram: sexo, idade, procedência, tempo de espera para classificação de risco, especialidade médica e desfecho após atendimento médico (Tabela 4). Ao analisar a relação entre essas variáveis e a prioridade de atendimento dos pacientes de acordo com o protocolo de Manchester, a metodologia bivariada evidenciou que os pacientes do sexo feminino (valor $p=$ 0,014 ) apresentaram uma maior chance de classificação de risco em baixa prioridade, mesmo comportamento dos idosos com idade menor ou igual a 70 anos (valor $p=0,026$ ).

A classificação de alta prioridade é $65 \%$ menor entre as mulheres e $44 \%$ menor entre os pacientes com idade entre 60 e 70 anos, conforme valores do Odds Ratio (OR) na Tabela 4. A estatística OR é uma medida de força da associação constatada pelo teste Qui-Quadrado, já mencionado.

Em relação à procedência (valor $p=0,0001$ ) os pacientes que se dirigiram ao serviço de saúde espontaneamente tiveram maior chance de classificação de risco em baixa prioridade, $82 \%$ menor que os pacientes encaminhados ao serviço (centro de saúde, SAMU e UPA).

Os pacientes que esperaram tempo maior que 10 minutos para classificação de risco (valor $p=0,04$ ), tiveram maior chance de esta ser em baixa prioridade (duas vezes maior que os atendidos em até 10 minutos), em consonância com o preconizado no protocolo de Manchester. Os pacientes atendidos pela especialidade 
clínica médica (valor $p=0,011$ ) tiveram maior chance de classificação de risco em alta prioridade, pois os pacientes atendidos pela cirurgia geral tiveram $81 \%$ menos chances de terem esta classificação. Já os pacientes que após atendimento médico evoluíram para alta domiciliar tiveram maior chance de classificação de risco em baixa prioridade ( $76 \%$ menor que os que tiveram como desfecho do atendimento a transferência)

(Tabela 4).

Tabela 4 - Variáveis avaliadas em relação a gravidade. MG - 2018.

\begin{tabular}{|c|c|c|c|c|c|c|}
\hline \multirow{2}{*}{ Variáveis } & \multicolumn{2}{|c|}{$\begin{array}{c}\text { Baixa } \\
\text { Prioridade }\end{array}$} & \multicolumn{2}{|c|}{$\begin{array}{c}\text { Alta } \\
\text { Prioridade }\end{array}$} & \multirow[t]{2}{*}{ OR } & \multirow{2}{*}{ p-valor } \\
\hline & $\mathbf{n}$ & $\%$ & $\mathbf{n}$ & $\%$ & & \\
\hline \multicolumn{7}{|l|}{ Sexo } \\
\hline Feminino & 213 & 63,8 & 18 & 43,9 & $0,445(0,231-0,857)$ & \multirow{2}{*}{$\mathrm{p}=0,014$} \\
\hline Masculino & 121 & 36,2 & 23 & 56,1 & 1,0 (ref) & \\
\hline \multicolumn{7}{|l|}{ Regional } \\
\hline Noroeste & 99 & 30 & 15 & 36,6 & $1,346(0,684-2,651)$ & \multirow{2}{*}{$\mathrm{p}=0,389$} \\
\hline Outras & 231 & 70 & 26 & 63,4 & 1,0 (ref) & \\
\hline \multicolumn{7}{|l|}{ Cidade } \\
\hline $\mathrm{BH}$ & 297 & 88,9 & 36 & 87,8 & $0,897(0,331-2,428)$ & \multirow{2}{*}{$\mathrm{p}=0,83$} \\
\hline Região Metropolitana & 37 & 11,1 & 5 & 12,2 & 1,0 (ref) & \\
\hline \multicolumn{7}{|l|}{ Faixa etária } \\
\hline Até 70 anos & 183 & 54,8 & 15 & 36,6 & $0,656(0,282-1,522)$ & \multirow{3}{*}{$\mathrm{p}=0,026$} \\
\hline Entre 71 e 80 anos & 80 & 24,0 & 10 & 24,4 & $1,0($ ref $)$ & \\
\hline Acima de 80 anos & 71 & 21,3 & 16 & 39,0 & $0,555(0,237-1,301)$ & \\
\hline \multicolumn{7}{|l|}{ № de atendimentos } \\
\hline Nenhum & 70 & 21 & 5 & 12,2 & $0,458(0,162-1,297)$ & \multirow{3}{*}{$\mathrm{p}=0,325$} \\
\hline Até 2 & 109 & 32,6 & 17 & 41,5 & 1,0 (ref) & \\
\hline 3 ou mais & 155 & 46,4 & 19 & 46,3 & $1,272(0,633-2,559)$ & \\
\hline \multicolumn{7}{|l|}{$\begin{array}{l}\text { Tempo de espera - } \\
\text { classificação }\end{array}$} \\
\hline Até 10 minutos & 155 & 46,4 & 26 & 63,4 & $2,002(1,023-3,915)$ & \multirow{2}{*}{$p=0,04$} \\
\hline Acima de 10 minutos & 179 & 53,6 & 15 & 36,6 & 1,0 (ref) & \\
\hline \multicolumn{7}{|l|}{ Procedência } \\
\hline Demanda Espontânea & 316 & 94,6 & 31 & 75,6 & $0,177(0,075-0,416)$ & \multirow{2}{*}{$p=0,000$} \\
\hline Outras & 18 & 5,4 & 10 & 24,4 & 1,0 (ref) & \\
\hline \multicolumn{7}{|l|}{ Desvio } \\
\hline Cirurgia Geral & 34 & 10,1 & 1 & 2,4 & $0,186(0,025-1,4)$ & \multirow{3}{*}{$\mathrm{p}=0,011$} \\
\hline Cirurgia Médica & 247 & 74 & 39 & 95,2 & $1,0($ ref $)$ & \\
\hline Ortopedia & 53 & 15,9 & 1 & 2,4 & $8,368(1,125-62,268)$ & \\
\hline \multicolumn{7}{|l|}{ Desfecho } \\
\hline Alta para exterior & 299 & 89,5 & 25 & 61,0 & $0,237(0,109-0,514)$ & \multirow{3}{*}{$\mathrm{p}=0,000$} \\
\hline Alta por transferência & 34 & 10,2 & 12 & 29,3 & 1,0 (ref) & \\
\hline Óbito & 1 & 0,3 & 4 & 9,7 & $0,088(0,009-0,870)$ & \\
\hline
\end{tabular}

Fonte: Alkmim AKS e Santos CS, 2020.

\section{DISCUSSÃO}

Após análise dos dados, observou-se que o perfil sociodemográfico da maior proporção de pacientes que compuseram a amostra foi de mulheres, a idade média dos pacientes foi de 72,94 anos, mínimo de 60 e máxima de 99 anos. Outros estudos apresentam o mesmo perfil de atendimento e discorrem sobre o papel da mulher como protagonista do autocuidado em saúde (RÊGO AS, et al., 2017; SANTOS FC, 2018; SILVA NTF, et al., 2018). 
O fato de haver mais idosos do sexo feminino dentre a amostra em estudo corrobora com os apontamentos de Almeida AV, et al. (2015), sobre a feminização da velhice. De acordo com as autoras, as mulheres vivem em média sete anos mais que os homens e a população possuem proporção de mulheres maior em grupos etários mais velhos (ALMEIDA AV, et al., 2015).

Ao caracterizar o atendimento, verificou-se que a maioria dos registros foi de idosos procedentes da região onde a UPA se localiza, outros autores obtiveram resultados semelhantes com este dado, evidenciando que a procedência dos usuários das UPA segue a lógica da proximidade geográfica, isto é, os usuários utilizam as UPA que estão mais próximas de sua residência, buscando a unidade localizada em sua regional (CASSETTARI SSR e MELLO ALSF, 2017; PÍCOLI RP, et al., 2016).

Sobre o perfil clínico dos pacientes que compuseram a amostra, as principais comorbidades encontradas na população estudada foram hipertensão arterial $(46,0 \%)$ e diabetes $(25,0 \%)$, próximas aos resultados encontrados na literatura. Resultado semelhante foi encontrado, em um estudo realizado em São Paulo, que observou a presença de comorbidades em idosos, variando de $55 \%$ a $98 \%$, semelhante ao público que a UPA Noroeste assiste (ALMEIDA AV, et al., 2015; OLIVEIRA CP, et al., 2018).

As comorbidades estão entre os principais problemas relacionados com a população que envelhece, sendo umas das principais causas que levam ao declínio funcional e risco de morte para a população idosa. A mudança no perfil populacional dos países que têm envelhecido rapidamente prevê o aumento de doenças crônicas não transmissíveis (BRASIL, 2012). Essa mudança de perfil populacional demanda organização dos serviços de saúde para atender essa realidade, o que leva a refletir sobre a importância do acompanhamento da população idosa pela Atenção Primária.

A principal queixa referida pelos usuários desse estudo foi problemas nos membros corroborando com estudo realizado em um pronto-socorro de um hospital de grande porte da cidade de Belo Horizonte, Minas Gerais (CHIANCA TCM, et al., 2016). Vale ressaltar que as principais queixas (problemas nos membros, dor abdominal e mal-estar) integram os problemas passíveis de serem atendidos na atenção primária. Esta demanda é indicativa de um desarranjo da oferta de serviços, na atenção primária, na média e alta complexidade (RISSARDO LK, et al., 2016).

O fluxograma mais frequente neste estudo foi problemas de extremidades, tal achado reforça os resultados de estudos realizados em dois hospitais do estado de Minas Gerais, que revelaram alta prevalência do mesmo fluxograma (GUEDES HM et al., 2015; CHIANCA TCM, et al., 2016). O motivo que pode ter contribuído para esse resultado é a instituição ser considerada referência regional para o atendimento de demandas de cirurgia vascular, além de apresentar grande demanda de pacientes atendidos por problemas ortopédicos.

A produtividade da rede de urgência e emergência é relacionada a um AACR de qualidade, consequentemente é possível analisar se a procura pelo serviço de atendimento era realmente necessária (GUEDES HM, et al., 2014). Na presente pesquisa, observou-se que a maioria dos pacientes procurou atendimento por demanda espontânea e foram classificados com a cor verde/azul $(53,1 \%)$ destinada a situações pouco urgentes.

Muitos estudos discutem a real necessidade dos atendimentos não urgentes em UPA, sendo que esta demanda poderia ser resolvida na APS. Um estudo realizado por Becker JB, et al. (2015) analisou a classificação do paciente por cinco prioridades de atendimento: vermelho, laranja, amarelo, verde e azul, em um Hospital Escola Federal, na cidade de São Paulo, no qual 73,7\% foram classificados nas cores verde ou azul. Outro estudo desenvolvido na cidade de São Paulo observou que $61,0 \%$ dos pacientes que procuram a unidade receberam a classificação verde (OLIVEIRA GN, et al., 2016).

Booker MJ, et al. (2015), por meio de uma revisão sistemática desenvolvida para entender os fatores que levam a população a buscar serviços de emergência, quando estes poderiam ser atendidos na atenção primária, evidenciaram que características individuais dos pacientes e dos seus cuidadores, aspectos relacionados à infraestrutura e organização dos serviços, bem como fatores sócio populacionais influenciam a decisão de procurar por este tipo de serviço. Assim, a escolha dos serviços pelo idoso é influenciada principalmente pela gravidade ou urgência do problema, estrutura disponível, resolubilidade, acolhimento, distância dos serviços de saúde e agilidade no atendimento (BRASIL, 2002). 
O vínculo longitudinal é uma das particularidades da APS e tem como fundamento o conhecimento das necessidades de saúde da população, favorecendo a utilização da unidade básica de saúde como fonte contínua de cuidado para os vários episódios de doença e cuidados preventivos. Algumas ações na APS como prevenção, diagnóstico, realização do acompanhamento e controle da saúde de pessoas com doenças crônicas e educação em saúde, quando efetivas, favorecem o vínculo, bem como a conscientização da população sobre a utilização correta dos níveis de atenção, reduzindo a demanda de atendimentos sensíveis à atenção primária em outros níveis de complexidade (RÊGO AS, et al., 2017).

Quanto aos desfechos após atendimento médico, mais da metade dos idosos recebeu alta após consulta/medicação. Pesquisa realizada no estado de São Paulo mostrou resultado semelhante, em que $94,5 \%$ dos pacientes exibiram o mesmo desfecho e, em sua maioria, foram classificados em níveis de prioridade de menor gravidade (SCOLARI GAS, et al., 2018).

No desfecho, relacionando as variáveis à prioridade do atendimento, a metodologia bivariada evidenciou que os pacientes do sexo feminino (valor $p=0,014$ ) apresentaram uma maior chance de classificação de risco em baixa prioridade. A associação observada vai ao encontro de muitos estudos publicados, visto que o sexo feminino foi um fator preditor de maior busca por assistência à saúde (LEVORATO CD, et al., 2014; RISSARDO LK, et al., 2016).

Pesquisas indicam que, no Brasil, as mulheres utilizam mais os serviços de saúde, uma justificativa considerável seria a baixa adesão dos homens à prevenção e maior exposição a fatores de risco durante a vida, sendo que essas diferenças são determinantes do consumo pelos serviços entre os sexos, dado semelhante ao evidenciado no presente estudo (BRASIL, 2010; LEVORATO CD, et al., 2014).

A associação entre a idade e a prioridade do atendimento revelou que a classificação em alta prioridade é $44 \%$ menor entre os pacientes com idade menor ou igual a 70 anos (valor $p=0,026$ ), conforme valores do Odds Ratio (OR) na Tabela 4. Estudos prévios apontam que as idades mais longevas é fator relevante para o adoecimento e a pior qualidade de vida (SANTOS FC, 2018; SILVA ADC, et al., 2019). Em estudo nacional que avaliou a demanda de um serviço de emergência utilizando o Protocolo Manchester, os resultados demonstraram que pacientes com maior idade foram classificados nas categorias de maior gravidade (DINIZ AS, et al., 2014).

Os pacientes que esperaram tempo maior que 10 minutos para classificação de risco (valor $p=0,04$ ), tiveram maior chance de esta ser em baixa prioridade (duas vezes maior que os atendidos em até 10 minutos), em consonância com o que está preconizado no protocolo de Manchester.

Recomenda-se que o tempo entre a chegada do paciente ao serviço e a classificação de risco não ultrapasse 10 minutos, para não causar dano, principalmente aos pacientes classificados com alto nível de gravidade (SILVA ADC, et al., 2019).

Vale ressaltar que, na prática clínica, o paciente classificado como alta prioridade é encaminhado diretamente para a sala de emergência, onde possui equipe assistencial disponível imediatamente para os atendimentos de urgência. Assim, os dados referentes ao tempo de atendimento para esses pacientes não estão relacionados à ausência de avaliação e assistência médica antes do registro do atendimento médico nas bases de dados do hospital (CHIANCA TCM, et al., 2016).

O cálculo da Odds Ratio demonstrou maior gravidade em pacientes atendidos na especialidade clínica médica (valor $p=0,011$ ). Resultados equivalentes foram encontrados em um estudo português que avaliou admissão hospitalar e mortalidade utilizando o Protocolo Manchester, com maior número de mortes e internação hospitalar associado principalmente nas especialidades clínicas (ZACHARIASSE JM, et al., 2017).

Os pacientes que após atendimento evoluíram para alta médica hospitalar (valor $p=0,000$ ) foram responsáveis por $86,4 \%$ dos atendimentos e destes, $92,3 \%$ foram classificados como baixa prioridade. Estes resultados apontam o uso inadequado do serviço de emergência, já que a maior parte dos pacientes que buscam as UPA poderiam ser atendidos em serviços de menor complexidade. A baixa resolutividade da atenção básica e a precariedade da rede hospitalar ocasionam a superlotação dos serviços de emergência do País (BECKER JB, et al., 2015). 
Um fato importante a ser especificado, por se tratar de um estudo com coleta de dados dos prontuários são as deficiências e dificuldades inerentes à fonte de dados. O preenchimento manual dos profissionais assistenciais está sujeito a erros de registro e/ou má qualidade. Espera-se que por meio da padronização da coleta de dados, prováveis vieses possam ser minimizados.

\section{CONCLUSÃO}

O levantamento das características sociodemográficas e clínicas da população atendida em serviços de urgência e emergência torna-se essencial para a definição de prioridades e o planejamento das ações de melhorias do serviço e da assistência em saúde. O estudo permitiu caracterizar a demanda de idosos e o tipo de atendimentos realizados na UPA Noroeste, em 2018. Foi possível identificar que uma parcela importante dos atendimentos se tratava de atendimentos não urgentes que em grande parte, poderiam ser atendidos e solucionados na APS, o que aponta uma descaracterização do verdadeiro papel das UPA, enfatizando a necessidade do fortalecimento da atenção primária como porta de entrada, com mecanismos que esclareçam aos usuários as atribuições de cada ponto da rede.

\section{REFERÊNCIAS}

1. ALMEIDA AV, et al. A feminização da velhice: em foco as características socioeconômicas, pessoais e familiares das idosas e o risco social. Textos \& Contextos, 2015; $14(1): 115-131$.

2. ANDRADE LAS, et al. Cuidado do idoso no setor de emergência: uma revisão integrativa. Rev. Bras. Geriat. Gerontol., 2018; 21(2): 249-260.

3. BECKER JB, et al. Triagem no serviço de emergência: associação entre as suas categorias e os desfechos do paciente. Rev. Esc. Enferm., 2015; 49(5):783-789.

4. BOOKER MJ, SHAW ARG, PURDY S. Why do patients with 'primary care sensitive' problems access ambulance services? A systematic mapping review of the literature. BMJ Open, 2015; 5(5): e007726.

5. BRASIL. Ministério da Saúde. Portaria GM n. 2.048, de 5 de novembro de 2002.

6. BRASIL. Instituto Brasileiro de Geografia e Estatística (IBGE). Síntese de indicadores sociais: uma análise das condições de vida da população brasileira; 2010. Rio de Janeiro: IBGE; 2010. 2014.

7. BRASIL. Ministério da Saúde. Organização Pan-Americana da Saúde. As redes de atenção à saúde. Brasília, 2011. 549 p.

8. BRASIL. Ministério da Saúde. Secretaria de Atenção à Saúde. Departamento de Atenção Básica. Política Nacional de Atencão Básica. Brasília: Ministério da Saúde, 2012.

9. BRASSIL. Ministério da Saúde. Secretaria de Atenção à Saúde. Departamento de Atenção Especializada. Manual instrutivo da Rede de Atenção às Urgências e Emergências no Sistema Unico de Saúde (SUS). Brasília: Ministério da Saúde, 2013. $84 \mathrm{p}$.

10. CASSETTARI, SSR, MELLO ALSF. Demanda e tipo de atendimento realizado em unidades de pronto atendimento do município de Florianópolis, Brasil. Texto \& Contexto Enferm., 2017; 26(1):e3400015.

11. CHIANCA TCM, et al. Tempos de espera para atendimento usando Sistema de Triagem de Manchester em um hospital de urgência. REME - Rev. Min. Enferm., 2016; 20:e988.

12. DINIZ AS, et al. Demanda clínica de uma unidade de pronto atendimento, segundo o protocolo de Manchester. Rev. Eletr. Enf., 2014; 16(2):312-20.

13. GONÇALES PC, et al. Relação entre estratificação de risco, mortalidade e tempo de permanência em um hospital de urgência. Invest. Educ. Enferm., 2015; 33(3):424-431.

14. GUEDES HM, et al. Classificação de risco: retrato de população atendida num serviço de urgência brasileiro. Rev. Enf. Ref., 2014; 5(1):37-44.

15. GUEDES HM, MARTINS JCA, CHIANCA TCM. Valor de predição do Sistema de Triagem de Manchester: avaliação dos desfechos clínicos de paciente. Rev. Bras. Enferm., 2015; 68(1):45-51.

16. LEVORATO CD, et al. Fatores associados à procura por serviços de saúde numa perspectiva relacional de gênero. Ciênc. Saúde Coletiva, 2014; $19(4): 1263-1274$.

17. MARCONATO RS, MONTEIRO MI. Prioridades da classificação de risco em uma unidade de emergência e desfecho do

18. OLIVEIRA GN, et al. Correlação das categorias de classificação de risco com aspectos clínicos e desfechos. Rev Latino-Am Enfermagem, 2016; 24:e2842.

19. OLIVEIRA CP, et al. Perfil epidemiológico de pacientes idosos atendidos em um pronto-socorro de hospital universitário brasileiro. Revista de Medicina, 2018; $97(1): 44-50$.

20. PÍCOLI RP, CAZOLA LHO, MAURER NMJS. Usuários de classificação de risco azul em uma unidade de pronto atendimento. Cogitare Enfermagem, 2016; 21(1):01-07.

21. REGO AS, et al. Fatores associados ao atendimento a idosos por condições sensíveis à Atenção Primária à Saúde. Rev. Bras. Geriatr. Gerontol. 2017; 20(6): 778-789.

22. RISSARDO LK, et al. Idosos atendidos em unidade de pronto-atendimento por condições sensíveis à Atenção Primária à Saúde. REME - Rev. Min. Enferm., 2016; 20:e971.

23. SANTOS FC. Qualidade de vida e fatores associados segundo os determinantes sociais da saúde em idosos: estudo transversal de base populacional. Dissertacão (Mestrado em Enfermagem) - Escola de Enfermagem da Universidade Federal de Minas Gerais, Belo Horizonte 2018; 118p.

24. SANTOS RC. Violência e fragilidade em idosos atendidos numa unidade de pronto atendimento. Dissertação (Mestrado em Enfermagem) - Universidade Federal do Rio Grande do Norte, Natal 2017; 108p.

25. SCOLARI GAS, RISSARDO LK, CARREIRA L. Produção científica sobre idosos em unidades de pronto atendimento. Rev. Enferm. UFPE, 2018;12(2):520-530.

26. SILVA ADC, et al. Caracterizacão dos atendimentos de um pronto-socorro público segundo o Sistema de Triagem de Manchester. REME - Rev. Min. Enferm., 2019;23: e-1178.

27. SILVA NTF, et al. Perfil de idosos, vítimas de trauma, atendidos em unidade de pronto atendimento de um hospital de ensino. Ciência, Cuidado e Saúde, 2018; 17(2):1-8.

28. TIENSOLI SD, et al. Características dos idosos atendidos em um pronto-socorro em decorrência de queda. Rev. Gaúcha Enferm., 2019; 40: e20180285.

29. ZACHARIASSE JM, et al. Validade do sistema de triagem de Manchester em atendimento de emergência: um estudo observacional prospectivo. 2017. 